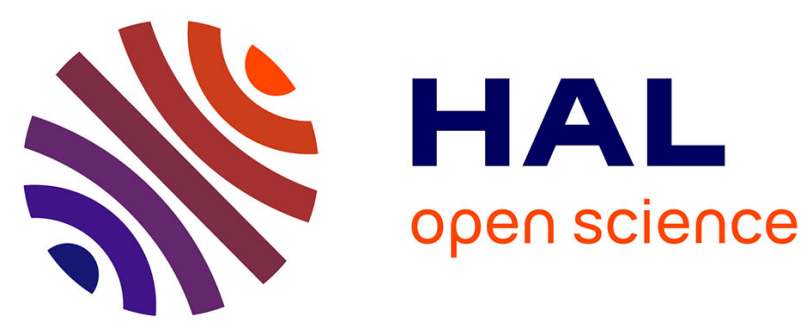

\title{
Multilabel, multiscale topological transformation for cerebral MRI segmentation post-processing
}

\author{
Carlos Tor-Díez, Sylvain Faisan, Loïc Mazo, Nathalie Bednarek, Hélène
}

Meunier, Isabelle Bloch, Nicolas Passat, François Rousseau

\section{- To cite this version:}

Carlos Tor-Díez, Sylvain Faisan, Loïc Mazo, Nathalie Bednarek, Hélène Meunier, et al.. Multilabel, multiscale topological transformation for cerebral MRI segmentation post-processing. International Symposium on Mathematical Morphology (ISMM), 2019, Saarbrücken, Germany. pp.471-482, 10.1007/978-3-030-20867-7_36 . hal-01982972

\section{HAL Id: hal-01982972 \\ https://hal.science/hal-01982972}

Submitted on 23 Mar 2019

HAL is a multi-disciplinary open access archive for the deposit and dissemination of scientific research documents, whether they are published or not. The documents may come from teaching and research institutions in France or abroad, or from public or private research centers.
L'archive ouverte pluridisciplinaire $\mathbf{H A L}$, est destinée au dépôt et à la diffusion de documents scientifiques de niveau recherche, publiés ou non, émanant des établissements d'enseignement et de recherche français ou étrangers, des laboratoires publics ou privés. 


\title{
Multilabel, Multiscale Topological Transformation for Cerebral MRI Segmentation Post-processing
}

\author{
Carlos Tor-Díez ${ }^{1}$, Sylvain Faisan ${ }^{2}$, Loïc Mazo ${ }^{2}$, Nathalie Bednarek ${ }^{3,4}$, \\ Hélène Meunier ${ }^{4}$, Isabelle Bloch ${ }^{5}$, Nicolas Passat ${ }^{3}$, and François Rousseau ${ }^{1}$ \\ 1 IMT Atlantique, LaTIM U1101 INSERM, UBL, Brest, France \\ 2 ICube UMR 7357, Université de Strasbourg, CNRS, FMTS, Illkirch, France \\ 3 Université de Reims Champagne-Ardenne, CReSTIC, Reims, France \\ 4 Service de médecine néonatale et réanimation pédiatrique, CHU de Reims, France \\ ${ }^{5}$ LTCI, Télécom ParisTech, Université Paris-Saclay, Paris, France
}

\begin{abstract}
Accurate segmentation of cerebral structures remains, after two decades of research, a complex task. In particular, obtaining satisfactory results in terms of topology, in addition to quantitative and geometrically correct properties is still an ongoing issue. In this paper, we investigate how recent advances in multilabel topology and homotopytype preserving transformations can be involved in the development of multiscale topological modelling of brain structures, and topology-based post-processing of segmentation maps of brain MR images. In this context, a preliminary study and a proof-of-concept are presented.
\end{abstract}

Keywords: Homotopic deformation $\cdot$ Multilabel topology $\cdot$ Multiscale modelling $\cdot$ Segmentation $\cdot$ MRI $\cdot$ Brain

\section{Introduction}

Topological methods generate a growing interest in the field of medical image processing and analysis. Indeed, ensuring the coherence of structural properties of the organs and tissues in 3D medical data is a cornerstone e.g. for registration, modelling or visualization tasks. In particular, topological concepts developed in the field of discrete imagery, and in particular digital topology, can allow for the development of efficient approaches that take into account not only quantitative and morphometric information carried by anatomical objects of interest, but also more intrinsic properties related to their structure [21].

In particular, the brain has received a specific attention. Indeed, by contrast with other organs, it exhibits an important interindividual variability from shape and size points of view. In the meantime, it is organized into many distinct subparts and tissues with a strong topological invariance. Taking into account topological priors is then a relevant hypothesis for guiding and/or regularizing image processing procedures [19].

This article presents a contribution in the field of topology-based brain structure segmentation. More especially, we focus on topological post-processing of 
multi-label segmentation maps obtained beforehand with efficient, but nontopologically guided, methods. Our purpose is then to build, from such segmentation maps, a corrected output consolidated by topological priors.

This research area is not new; related works are recalled in Section 2. However, the embedding of topological priors in segmentation paradigms is a complex task, a fortiori when we consider $n$-ary segmentation, with $n>2$, i.e. more than one object vs. its background. In this context, our contributions are the following. First, we rely on a topological framework introduced a few years ago by some of the authors, that allows to correctly model digital images by considering the topology of $n$ labels but also that of the combinations within their power lattice $[18,16]$. Second, we consider a multiscale approach for topological modelling of the cerebral structures. Indeed, we assume that the topological assumptions that should guide a segmentation process actually depend on the level of details of the observed structures. Based on these two, multilabel and multiscale, paradigms, we develop a generic, homotopic deformable model methodology that progressively refines a segmentation map with respect to the data and the associated topological priors.

In Section 3, we recall the involved topological framework and how it can be used for multiscale, multilabel topological modelling of anatomical structures. In Section 4, we describe the algorithmic scheme that allows us to progressively refine an initial segmentation with respect to this multiscale topological modelling. Experimental results are proposed in Section 5; at this stage, they have mainly an illustrative value. Section 6 concludes this article by presenting the main perspectives offered by the proposed approach and the remaining challenges to be tackled.

\section{Related Works}

Topology-based segmentation methods dedicated to brain structures can be mainly divided into two families [19]: on the one hand, the methods that consider a topological prior for guidance from the very beginning of the process; on the other hand, the methods that aim at recovering a posteriori some correct topological properties. The first lie in the family of topological deformable models; the second in the family of topological correction methods.

In most of these methods, the considered structures of interest are the main three classes of tissues, namely grey matter (GM) (mainly, the cortex), white matter (WM), and cerebrospinal fluid (CSF). A majority of the proposed methods focus on the cortex, that presents a complex geometry, with strong folding and a low thickness, leading to a high curvature 2D-like thin ribbon. Cortex segmentation is generally presented as a binary segmentation problem (cortex vs. other structures) or a ternary segmentation problem (GM, WM, CSF). The associated topological hypotheses are often a simplified version of the anatomical reality, and the different classes of tissues are assumed nested: the central class is simply connected (i.e. a full sphere) with successively nested hollow spheres. Based on these simplified assumptions, it is possible to develop segmentation 
strategies from binary digital topology [13], and in particular homotopic transformations based on simple points [8] (less frequently, alternative topological models were proposed, for instance cellular complexes $[7,9]$ ).

Since the pioneering works proposed in [15], different variants of such topological deformable models have been proposed, in fully discrete paradigms [5] or by coupling continuous and digital models [11]. However, the hard topological constraints imposed by the model can lead to deadlocks that are difficult to handle (see [10] for a discussion on that topic). Based on the same topological hypotheses, many topology correction methods have been proposed for the cortex. They mainly consist of identifying the tunnels / handles generated on the cortical surface, and removing them based on ad hoc strategies [14,3]. These methods aim at reformulating the topological problem to be solved by considering a binary, simply connected topological model, which has the virtue to be easy to handle, but the drawback of poorly modelling the anatomical structures.

More recently, new ways were explored for tackling the issue of real multilabel segmentation and/or potentially complex topologies. Handling complex topology can be done by relying on less constraining - but also less robusttopological invariants, such as the genus [22]. Homotopic transformations based on simple points however remain the gold standard for carrying out model transformation. In order to avoid topological deadlocks, non-monotonic transformation processes of complex, multilabel topological models were investigated $[20,4]$. Although promising, these approaches suffer from various theoretical weaknesses $[20,2,23]$ (see [18] for a discussion). They also work at a unique scale, with induced difficulties to ensure simultaneously spatial / geometrical and topological reliability.

\section{Multilabel, Multiscale Topological Modelling}

\subsection{Theory}

In [16], Mazo proposed a framework for modelling the topology of multilabel digital images. The main ideas of this framework are as follows.

1. The multilabel image is splitted in a collection of binary images such that each binary image represents a region of interest, that is a region that has been previously labelled or represents a meaningful union of some labeled regions. The unions are labeled thanks to a lattice structure: the label of a union of regions is the supremum of the labels of the regions.

2. Each binary digital image is embeded in a partially ordered set (poset) by adding intervoxels elements (pointels, linels, surfels). Such elements are assigned to the foreground or the background thanks to the minimum (6adjacency) or maximum (26-adjacency) membership rule. Doing so, the connected components are preserved and the digital fundamental groups, as defined by Kong [12], are mapped, through isomorphisms, to the fundamental groups of the Alexandrov topology [17]. 


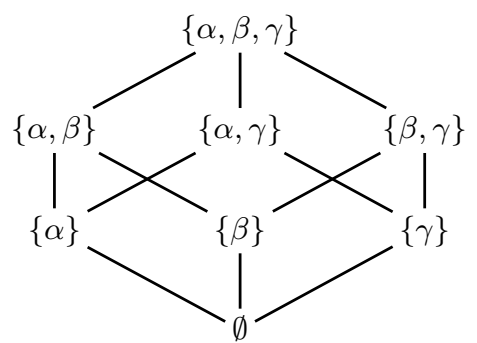

Fig. 1. The power lattice $\Lambda=2^{L}$ for the set of labels $L=\{\alpha, \beta, \gamma\}$ of the three considered classes of cerebral tissues.

In this framework, a homotopic relabelling boils down to a collection of simple point moves in binary images with the guarantee to preserve the underlying topological structures of the involved posets, and in particular their homotopytypes.

\subsection{Application}

In the applicative context of this work, our purpose is to post-process ternary classification maps of MR images defined as

$$
\begin{aligned}
F: \Omega & \rightarrow[0,1]^{3} \\
x & \mapsto\left(p_{\mathrm{CSF}}, p_{\mathrm{GM}}, p_{\mathrm{WM}}\right)
\end{aligned}
$$

where $\Omega$ is a part of $\mathbb{Z}^{3}$ corresponding to the support of an MR image, and for each point $x, p_{\mathrm{CSF}}, p_{\mathrm{GM}}$ and $p_{\mathrm{WM}}$ are the probabilities that $x$ belong to the cerebrospinal fluid, the grey matter (cortex) and the white matter, respectively. In particular, we have $\sum_{\ell} p_{\ell}=1$.

Such maps $F$ are obtained by a fuzzy segmentation process described in [24] (but they may be obtained via any other similar segmentation procedure, e.g. [6]). A specificity of this segmentation strategy — and many others - despite its good accuracy from quantitative and geometric points of view, lies in the fact that it is not guided by topological constraints. As a consequence, its output needs to be post-processed for any further application requiring topological guarantees (for instance mesh generation or differential cortical surface analysis).

Our purpose is then to build, from $F$, a new crisp segmentation map

$$
\begin{aligned}
T: \Omega & \rightarrow\{0,1\}^{3} \\
x & \mapsto\left(c_{C S F}, c_{G M}, c_{W M}\right)
\end{aligned}
$$

such that for each point $x, c_{C S F}, c_{G M}$ and $c_{W M}$ are equal to either 0 or 1 depending on the (unique) class of $x$, i.e. with $\sum_{\ell} c_{\ell}=1$.

In addition, $T$ should satisfy - unlike $F$ - some topological priors related to the structure of the different tissues. 


\subsection{Multiscale topological modelling}

The framework proposed in [16] allows us to model the topological structure of the three classes of cerebral tissues of interest, namely CSF, GM and WM, further noted $\gamma, \beta$ and $\alpha$, respectively, for the sake of concision. It also allows us to model any combinations of these labels, leading to the whole power lattice $\Lambda=2^{L} ;$ see Figure 1.

In particular, in [16], the notion of topology preservation relies on the definition of simple points that preserve the homotopy-type of all the labels of $\Lambda$. However, it is possible to consider the notion of simpleness for only a given (strict) subset of labels of $\Lambda$. In such a case, it is sufficient to guarantee that the relabelling of a (simple) point fulfills the required topological conditions for the chosen subset of labels, while the topology of the objects / complexes induced by the other labels are allowed to evolve.

This strategy, that only focuses on specific (combinations of) labels of interest, permits to define a multiscale topological modelling. Indeed, the notion of topology in discrete imaging is strongly related to the scale of observation of the objects of interest. For instance, fine topological details that are relevant at a high resolution, become useless (and sometimes incorrect) at a coarser resolution, due to the loss of precision induced by partial volume effects.

Based on this assumption, we consider two distinct topological models of the brain tissues, according to their scale (see Figure 2):

- Model 1 - Coarse / intermediate scales $\left(\mathbf{S}_{1}\right.$ and $\left.\mathbf{S}_{2}\right)$ : At these scales, we assume that the WM, GM and CSF are successively nested, which is the hypothesis currently considered in the literature. In other words, we aim at preserving the homotopy-type of three labels: $\{\alpha\}$ (simply connected); $\{\beta\}$ (hollow sphere); and $\gamma$ (hollow sphere); but also the homotopy-type of their four combinations.

- Model 2 - intermediate / fine scales $\left(\mathbf{S}_{2}\right.$ and $\left.\mathbf{S}_{3}\right)$ : At these scales, we assume that the cortex (GM) is no longer a hollow sphere. Indeed, on the lower part of the encephalus, the hypothesis of GM surrounding WM is not satisfied (due to the connection of the encephalus to the brainstem). Based on this hypothesis, we aim at preserving the homotopy-type of five labels: $\{\alpha\}$ (simply connected); $\{\alpha, \beta\}$ (simply connected); $\{\beta, \gamma\}$ (hollow sphere); $\{\gamma\}$ (hollow sphere); and $\{\alpha, \beta, \gamma\}$ (simply connected). In particular, this allows to make the topology of the cortex evolve whereas remaining coherent with respect to its neighbouring structures (CSF and WM).

\section{Multilabel, Multiscale Topology-Controlled Deformation}

We now describe how the topological assumptions modeled above can be considered for designing, at each scale, well-fitted homotopy-type preserving deformation processes. 


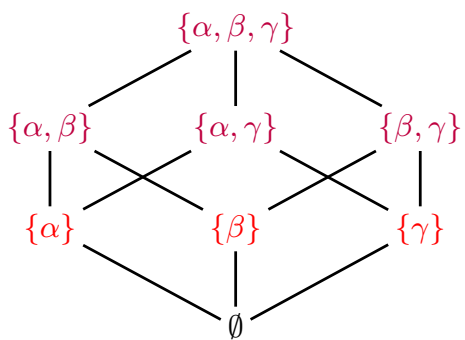

(a) Model 1: scales $1 \& 2$

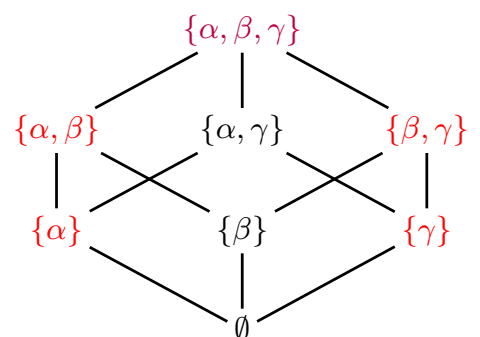

(b) Model 2: scales $2 \& 3$

Fig. 2. Topological modelling of the classes of brain tissues, at the coarse / intermediate (a) and intermediate / fine scales (b). The labels coloured in red and purple are those for which the topology has to be preserved. The red labels need to be explicitly handled; the topology of the purple labels is preserved as a corollary of the topological preservation of the red ones. The topology of the black labels is authorized to evolve during the transformation process.

\subsection{Grid refinement}

We work on a space $\Omega$ defined as a subset of $\mathbb{Z}^{3}$. In order to carry out the deformation process at each scale, the space $\Omega$ has to be adapted to the current scale. It is important to note that the notion of simple points and homotopy-type are compliant with respect to digital grid refinement. In other words, a digital object $X$ defined in $\mathbb{Z}^{3}$ (and more generally in $\mathbb{Z}^{n}$ ) has the same homotopy-type as its up-sampled analogue $X_{2}$ in $\mathbb{Z}^{3}$, defined by $x \in X_{2} \subset \mathbb{Z}^{3} \Leftrightarrow\lfloor x / 2\rfloor \in X$. In addition, $x$ is a simple point for $X$ iff there exists a sequence of successively simple points for $X_{2}$ composed from the 8 points of $2 x+\{0,1\}^{3}$. This topologypreserving octree refinement [1] remains trivially valid for up-samplings at any other (discrete) resolution.

In particular, we consider a scale factor $k \in \mathbb{N}, k>1$, between the grids of each scale. The set $\Gamma_{1} \subset \mathbb{Z}^{3}$ is the grid considered at the coarse scale $\mathrm{S}_{1}$. It is refined, at scale $\mathrm{S}_{2}$ into a second grid $\Gamma_{2}$ such that $\Gamma_{1}=k \Gamma_{2}$, which means that one point of $\Gamma_{1}$ corresponds to $k^{3}$ points of $\Gamma_{2}$. Finally, $\Gamma_{2}$ is refined into a third grid $\Gamma_{3}$ such that $\Gamma_{2}=k \Gamma_{3}$; this means that one point of $\Gamma_{1}$ corresponds to $k^{3}$ points of $\Gamma_{2}$ and $k^{6}$ points of $\Gamma_{3}$. Note that we have $\Omega=\Gamma_{3}$. It is then convenient to assume (without loss of generality) that $\Omega$ is defined as a Cartesian product $\prod_{i=1}^{3} \llbracket 0, N_{i}-1 \rrbracket \subset \mathbb{Z}^{3}$ such that $N_{i}$ is a multiple of $k^{2}$ for any $i=1,2,3$.

\subsection{Metrics}

In order to guide the topology-controlled transformation process, we need to define metrics for assessing the error between the current (evolving) segmentation map and the target segmentation map. In particular, by minimizing a cost function associated to this error, our purpose is to make our segmentation map progressively converge onto the target, whereas correctly handling the topology. 
In this context, we consider two metrics. The first is a classification metric, noted $M_{d}$, defined by the classification error at each point of the image. The second, noted $M_{\Delta}$, is a distance-based metric, defined by the $L_{1}$ distance between the misclassified points and the corresponding, correctly classified region in the target image.

In order to build these two metrics, we first need to define fuzzy classification maps $F_{i}$ from $F$ (see Eq. (1)) at each scale $i(i=1,2,3)$. This is done with a standard mean policy:

$$
F_{i}(x)=\frac{1}{k^{3 .(3-i)}} \sum_{a=0}^{k^{(3-i)}-1} \sum_{b=0}^{k^{(3-i)}-1} \sum_{c=0}^{k^{(3-i)}-1} F\left(k^{(3-i)} x+(a, b, c)\right) .
$$

In particular, we have $F_{3}=F$.

For a given (crisp) segmentation map $C$, the first metric $M_{d}$ assessing the error between $C$ and $F_{i}$ is then defined as:

$$
M_{d}\left(C, F_{i}\right)=\sum_{x}\left\|C(x)-F_{i}(x)\right\|_{2} .
$$

For defining the second metric $M_{\Delta}$, we build crisp classification maps $G_{i}$ from the fuzzy maps $F_{i}$ by a standard majority voting process, i.e. for any $x$, we set $G_{i}(x)=\arg _{\{\alpha, \beta, \gamma\}} \max F_{i}(x)$

For a given (crisp) map $C$, the second metric $M_{\Delta}$ assessing the error between $C$ and $G_{i}$ is then defined as:

$$
M_{\Delta}\left(C, G_{i}\right)=\sum_{x} \Delta\left(x, G_{i}^{-1}(C(x))\right)
$$

where $\Delta(x, X)=\min _{y \in X}\|x-y\|_{1}$ is the $L_{1}$ distance between the point $x$ and the set $X$, whereas $G_{i}^{-1}(v)=\left\{x \mid G_{i}(x)=v\right\}$. In particular, we have $\Delta\left(x, G_{i}^{-1}(C(x))\right)=0$ iff $C(x)=G_{i}(x)$.

\subsection{Initialization and optimization process}

The process is iterative, and proceeds from the coarse scale $S_{1}$ up to the fine scale $S_{3}$. For each step, the current input segmentation map is the output of the previous step (possibly up-sampled, if we switch between $S_{i}$ and $S_{i+1}$ ). The only explicit initialization is then required for the very first step of the process. At this stage, we simply consider a three-layer nested sphere model, with a central simply connected full sphere of label $\alpha$ surrounded by a first hollow sphere of label $\beta$ and finally a second hollow sphere of label $\gamma$.

At each step of the optimization process, we consider either the metric $M_{d}$ or $M_{\Delta}$. In order to make $M_{d}$ decrease, we build a map that defines, for each point $x$ and each possible relabelling $\ell_{1} \rightarrow \ell_{2}$ (with $\ell_{1}$ the current label at $x$ ), the associated benefit with respect to $M_{d}$, namely $\left\|\ell_{2}-F_{i}(x)\right\|_{2}-\left\|\ell_{1}-F_{i}(x)\right\|_{2}$. Then, we iteratively carry out the relabelling of (simple) points with the maximal (non-negative) benefit, until stability. 


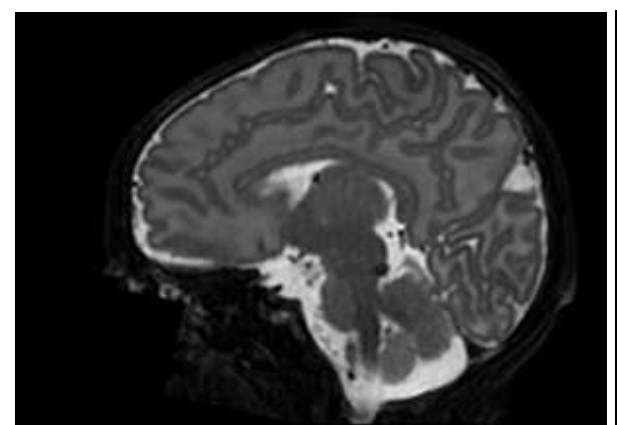

(a) $I$

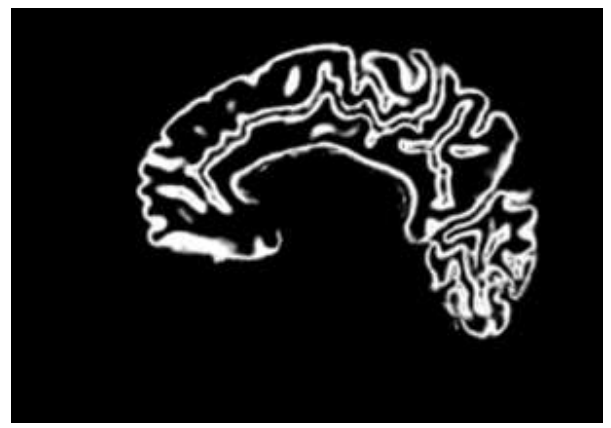

(c) $F_{G M}$

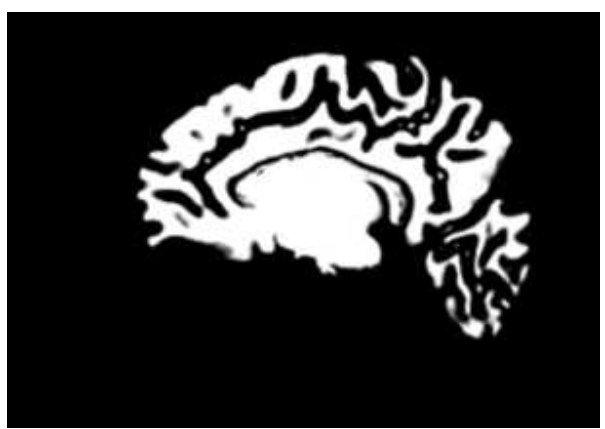

(b) $F_{W M}$

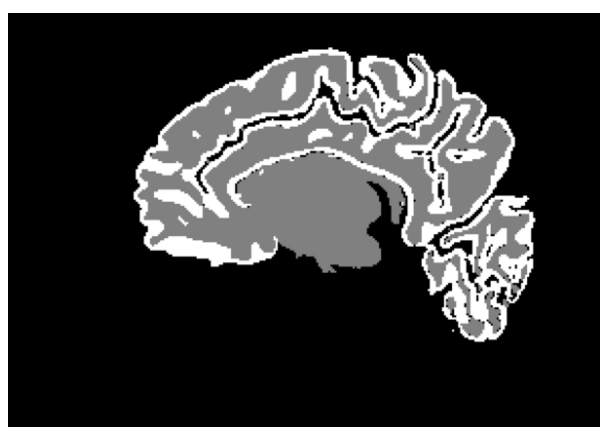

(d) $F$

Fig. 3. (a) MR image. (b) Fuzzy classification of the WM from (a). (c) Fuzzy classification of the GM from (a). (d) Crisp classification map obtained from the fuzzy classification maps of (a) (WM in grey; GM in white; CSF + background in black). (a-d) Sagittal slices.

In order to make $M_{\Delta}$ decrease, we build a map that defines, for each point $x$ and each possible relabelling $\ell_{1} \rightarrow \ell_{2}$ (with $\ell_{1}$ the current label at $x$ ), the associated benefit with respect to $M_{\Delta}$, as $\Delta\left(x, G_{i}^{-1}\left(\ell_{2}\right)\right)$ if $\Delta\left(x, G_{i}^{-1}\left(\ell_{2}\right)\right)<$ $\Delta\left(x, G_{i}^{-1}\left(\ell_{1}\right)\right)$ and 0 otherwise. Then, we iteratively carry out the relabelling of (simple) points with the maximal benefit, until stability.

\section{Experiments and Results}

We present some results computed with the proposed method, for topological correction of fuzzy segmentation maps obtained from the method proposed in [24] (or equivalent methods). An example of such map $F$ obtained from an MR image is illustrated in Figure 3.

Here, we carried out a 5 step iterative procedure with the meta-parameters summarized in Table 1.

The initial images $I$ are defined on $\Omega=\llbracket 0,291 \rrbracket \times \llbracket 0,291 \rrbracket \times \llbracket 0,203 \rrbracket$. We use as scale factor $k=2$. The successive segmentation maps (and the associated 
Table 1. Meta-parameters for the successive steps of the process.

\begin{tabular}{cccc}
\hline Step & Scale & Topology & Metric \\
\hline 1 & $\mathrm{~S}_{1}$ & Model 1 & $M_{\Delta}$ \\
2 & $\mathrm{~S}_{2}$ & Model 1 & $M_{\Delta}$ \\
3 & $\mathrm{~S}_{2}$ & Model 1 & $M_{d}$ \\
4 & $\mathrm{~S}_{2}$ & Model 2 & $M_{\Delta}$ \\
5 & $\mathrm{~S}_{3}$ & Model 2 & $M_{\Delta}$ \\
\hline
\end{tabular}

classification maps $F_{3}, F_{2}$ and $\left.F_{1}\right)$ are then defined on $\Gamma_{3}=\llbracket 0,291 \rrbracket \times \llbracket 0,291 \rrbracket \times$ $\llbracket 0,203 \rrbracket, \Gamma_{2}=\llbracket 0,145 \rrbracket \times \llbracket 0,145 \rrbracket \times \llbracket 0,101 \rrbracket$, and $\Gamma_{1}=\llbracket 0,72 \rrbracket \times \llbracket 0,72 \rrbracket \times \llbracket 0,50 \rrbracket$, respectively, i.e. with $\left|\Gamma_{3}\right| \simeq 1,7.10^{7},\left|\Gamma_{2}\right| \simeq 2,2.10^{6}$ and $\left|\Gamma_{1}\right| \simeq 2,7.10^{5}$.

An example of the successive steps of the topological transformation process is illustrated in Figure 4. One can observe the progressive convergence of the model toward the classification map, whereas controlling the topology of the segmentation, in particular on the cortex (preserved on the upper part of the encephalus), whereas the GM is relevantly removed on the lower part, in accordance with the initial classification map and with the topological model.

A 3D mesh visualization of the resulting map $T$ is illustrated in Figure 5, qualitatively emphasizing the topological correctness of the result.

\section{Conclusion}

This preliminary study provides a proof of concept for the relevance of using the multilabel topological framework proposed in [16] for developing a multiscale, topological modelling of the cerebral structures, allowing one to either preserve or relax topological constraints over the power lattice of a set of elementary semantic labels.

In particular, this framework can be efficiently used for carrying out multiscale, topology-controled deformation of label maps based on the concept of simple points, here in the context of topological correction of fuzzy segmentation maps computed beforehand.

Among numerous perspective works, we will further investigate (1) more sophisticated metrics for guiding the deformation process; (2) the possibility to carry out deformation processes at a superpixel resolution and/or to use cubical complex models [18] for topological modelling; and (3) a richer modelling of the brain with more anatomical structures, e.g. for atlas-based segmentation.

\section{Acknowledgements}

The research leading to these results has been supported by the ANR MAIA project $^{6}$, grant ANR-15-CE23-0009 of the French National Research Agency; INSERM and Institut Mines Télécom Atlantique (Chaire "Imagerie médicale en

\footnotetext{
${ }^{6}$ http://recherche.imt-atlantique.fr/maia
} 


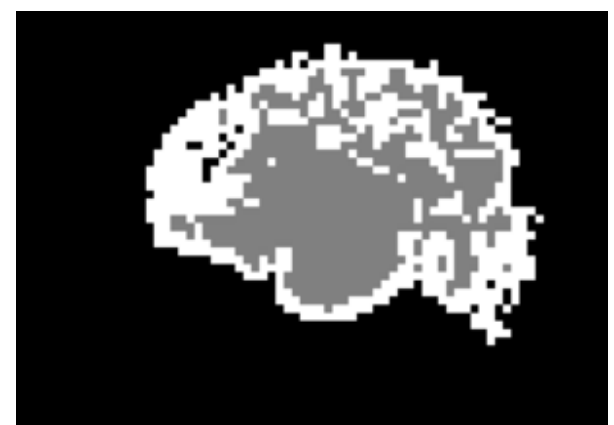

(a) Step 1

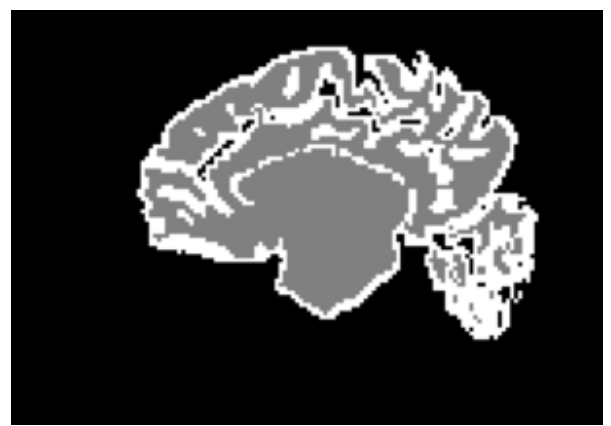

(c) Step 3

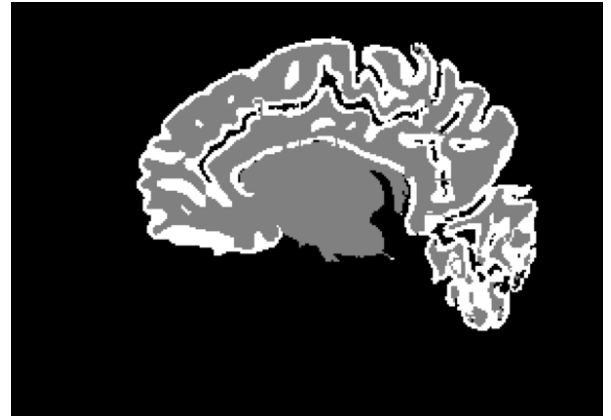

(e) Result $T$

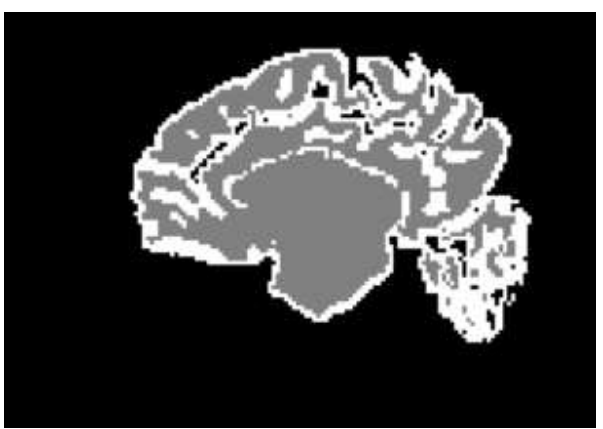

(b) Step 2

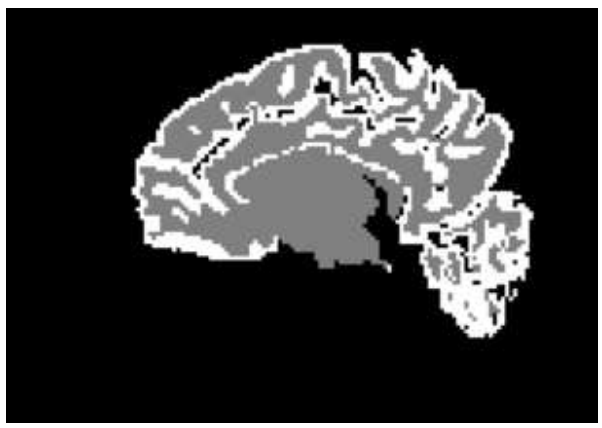

(d) Step 4

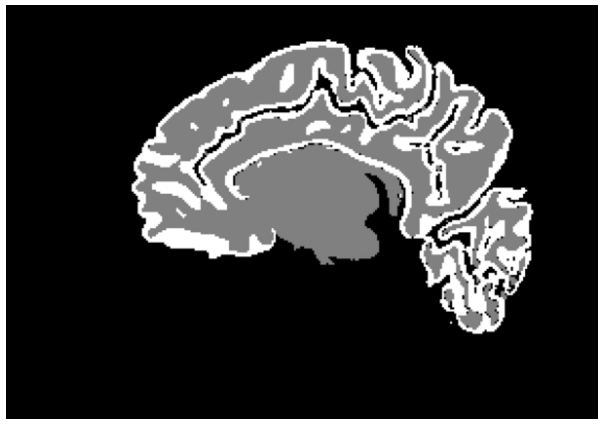

(f) $F$

Fig. 4. (a-d) Intermediate results obtained by the successive steps of the process (see Table 1). (e) Final segmentation map. (f) Crisp classification map (see also Figure 3(d)) used as reference for guiding the process.

thérapie interventionnelle"); the Fondation pour la Recherche Médicale (grant DIC20161236453); and the American Memorial Hospital Foundation. 


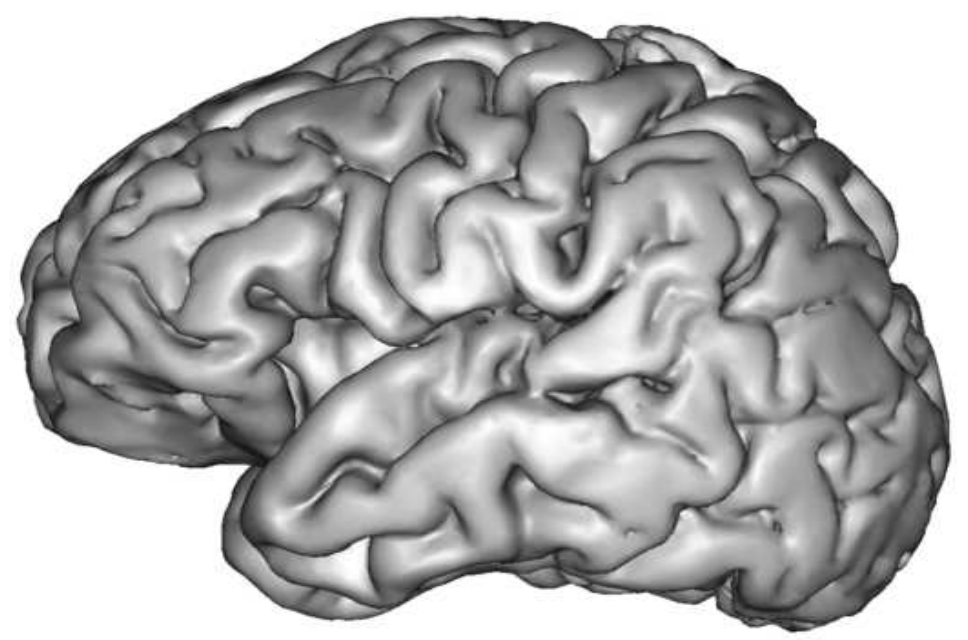

Fig. 5. Surface mesh generated from the final topological segmentation map $T$ of Figure $4(\mathrm{e})$.

\section{References}

1. Bai, Y., Han, X., Prince, J.L.: Digital topology on adaptive octree grids. Journal of Mathematical Imaging and Vision 34(2), 165-184 (2009)

2. Bazin, P.L., Ellingsen, L.M., Pham, D.L.: Digital homeomorphisms in deformable registration. In: Information Processing in Medical Imaging (IPMI), Proceedings. pp. 211-222 (2007)

3. Bazin, P.L., Pham, D.L.: Topology correction of segmented medical images using a fast marching algorithm. Computer Methods and Programs in Biomedicine 88(2), 182-190 (2007)

4. Bazin, P.L., Pham, D.L.: Topology-preserving tissue classification of magnetic resonance brain images. IEEE Transactions on Medical Imaging 26(4), 487-496 (2007)

5. Caldairou, B., Passat, N., Habas, P.A., Studholme, C., Koob, M., Dietemann, J.L., Rousseau, F.: Segmentation of the cortex in fetal MRI using a topological model. In: International Symposium on Biomedical Imaging (ISBI), Proceedings. pp. 2045-2048 (2011)

6. Caldairou, B., Passat, N., Habas, P.A., Studholme, C., Rousseau, F.: A non-local fuzzy segmentation method: Application to brain MRI. Pattern Recognition 44(9), 1916-1927 (2011)

7. Cointepas, Y., Bloch, I., Garnero, L.: A cellular model for multi-objects multidimensional homotopic deformations. Pattern Recognition 34(9), 1785-1798 (2001)

8. Couprie, M., Bertrand, G.: New characterizations of simple points in 2D, 3D, and $4 \mathrm{D}$ discrete spaces. IEEE Transactions on Pattern Analysis Machine Intelligence 31(4), 637-648 (2009)

9. Damiand, G., Dupas, A., Lachaud, J.O.: Fully deformable 3D digital partition model with topological control. Pattern Recognition Letters 32(9), 1374-1383 (2011) 
10. Faisan, S., Passat, N., Noblet, V., Chabrier, R., Meyer, C.: Topology preserving warping of 3-D binary images according to continuous one-to-one mappings. IEEE Transactions on Image Processing 20(8), 2135-2145 (2011)

11. Han, X., Xu, C., Prince, J.L.: A topology preserving level set method for geometric deformable models. IEEE Transactions on Pattern Analysis Machine Intelligence 25(6), 755-768 (2003)

12. Kong, T.Y.: A digital fundamental group. Computers \& Graphics 13(2), 159-166 (1989)

13. Kong, T.Y., Rosenfeld, A.: Digital topology: Introduction and survey. Computer Vision, Graphics, and Image Processing 48(3), 357-393 (1989)

14. Kriegeskorte, N., Goebel, N.: An efficient algorithm for topologically correct segmentation of the cortical sheet in anatomical MR volumes. NeuroImage 14(2), 329-346 (2001)

15. Mangin, J.F., Frouin, V., Bloch, I., Régis, J., López-Krahe, J.: From 3D magnetic resonance images to structural representations of the cortex topography using topology preserving deformations. Journal of Mathematical Imaging and Vision 5(4), 297-318 (1995)

16. Mazo, L.: A framework for label images. In: Computational Topology in Image Context (CTIC), Proceedings. pp. 1-10 (2012)

17. Mazo, L., Passat, N., Couprie, M., Ronse, C.: Digital imaging: A unified topological framework. Journal of Mathematical Imaging and Vision 44(1), 19-37 (2012)

18. Mazo, L., Passat, N., Couprie, M., Ronse, C.: Topology on digital label images. Journal of Mathematical Imaging and Vision 44(3), 254-281 (2012)

19. Pham, D.L., Bazin, P.L., Prince, J.L.: Digital topology in brain imaging. IEEE Signal Processing Magazine 27(4), 51-59 (2010)

20. Poupon, F., Mangin, J.F., Hasboun, D., Poupon, C., Magnin, I.E., Frouin, V.: Multi-object deformable templates dedicated to the segmentation of brain deep structures. In: Medical Image Computing and Computer-Assisted Intervention (MICCAI), Proceedings. pp. 1134-1143 (1998)

21. Saha, P.K., Strand, R., Borgefors, G.: Digital topology and geometry in medical imaging: A survey. IEEE Transactions on Medical Imaging 34(9), 1940-1964 (2015)

22. Ségonne, F.: Active contours under topology control - Genus preserving level sets. International Journal of Computer Vision 79(2), 107-117 (2008)

23. Siqueira, M., Latecki, L.J., Tustison, N.J., Gallier, J.H., Gee, J.C.: Topological repairing of 3D digital images. Journal of Mathematical Imaging and Vision 30(3), 249-274 (2008)

24. Tor-Díez, C., Passat, N., Bloch, I., Faisan, S., Bednarek, N., Rousseau, F.: An iterative multi-atlas patch-based approach for cortex segmentation from neonatal MRI. Computerized Medical Imaging and Graphics 70, 73-82 (2018) 